\title{
Control of multiple excited image states around segmented carbon nanotubes
}

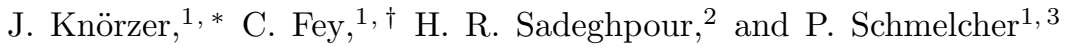 \\ ${ }^{1}$ Zentrum für Optische Quantentechnologien, Universität Hamburg, \\ Luruper Chaussee 149, 22761 Hamburg, Germany \\ ${ }^{2}$ ITAMP, Harvard-Smithsonian Center for Astrophysics, 60 Garden Street, Cambridge, MA 02138, USA \\ ${ }^{3}$ The Hamburg Centre for Ultrafast Imaging, Luruper Chaussee 149, 22761 Hamburg, Germany
}

(Dated: August 9, 2021)

\begin{abstract}
Electronic image states around segmented carbon nanotubes can be confined and shaped along the nanotube axis by engineering the image potential. We show how several such image states can be prepared simultaneously along the same nanotube. The inter-electronic distance can be controlled $a$ priori by engineering tubes of specific geometries. High sensitivity to external electric and magnetic fields can be exploited to manipulate these states and their mutual long-range interactions. These building blocks provide access to a new kind of tailored interacting quantum systems.
\end{abstract}

PACS numbers: 32.10.-f, 32.80.-t, 32.60.+i

\section{INTRODUCTION}

Image-potential states above metallic surfaces that arise from the interplay between an induced attractive image potential and a repulsive surface barrier have been of considerable interest in surface studies of conducting materials [1] 4]. Experimentally, these states can be observed with the aid of high-resolution time-resolved spectroscopy techniques [5] and their lifetimes were found to be on the order of tens of ps [6, 7. The energy levels of such states form a Rydberg series in the Coulomb-like image potential $V(z) \approx-\left[0.85 e^{2}(\varepsilon-1)\right] /[(\varepsilon+1) 4 z][8$ in a distance $z$ above the flat surface with dielectric constant $\varepsilon$.

In contrast, electronic states equipped with a non-zero angular momentum $l$ around infinitely long nanowires such as carbon nanotubes (CNTs) 9, or fullerenes [10, stem from the balance between image potential and centrifugal motion around nanostructure and are thus much more detached from the metallic surface. Tubular image states (TIS) surrounding CNTs were predicted some years ago, 9] and experimentally observed shortly afterward [11. High-angular momentum states with $\ell \gtrsim 5$ are fairly detached from the nanotube's surface and are predicted to possess comparatively long lifetimes of $\tau \approx 1 \mathrm{~ns}-1 \mu \mathrm{s}$ [12, but even low- $\ell$ TIS [13, have been shown to decay considerably slower than their counterparts above flat surfaces [1]. Extensive work has been done on Bloch states in lattices of CNTs aligned parallel [14 and periodically arranged finite segments combined by junctions constituting a single, long CNT [15. Furthermore, image-potential states in other systems [16, 17] have been considered in the context of quantum information processing. Since they are long-lived states and highly controllable by external fields [18, 19, Rydberglike TISs turn out to be interesting candidates for such purposes.

\footnotetext{
* e-mail: johannes.knoerzer@physnet.uni-hamburg.de

† e-mail: christian.fey@physnet.uni-hamburg.de
}

Our aim is to propose a scheme for the simultaneous preparation of multiple excited states around CNTs, allowing for subsequent studies of the long-range interactions in those systems. We consider single CNTs whose electronic properties give rise to confinement potentials along the longitudinal axis of the nanotube. Thereby, two external charges outside the CNT can be held at a controllable distance. We have found that specifically engineered CNTs in the presence of an external charge give rise to highly adjustable image potentials.

The present work is organized as follows: in Sec. III we describe the general properties of tubular image states around infinitely long nanowires. We address the question of how they are controllable in Sec. IIIA. Subsequently, in Sec. IIIB, the properties of finite and infinite geometries are interlinked. Sec. IIIC is dedicated to a general description of trapping two or more electrons in tubular image states around one segmented CNT, where the inter-electronic distance is, in principle, a priori controllable by geometry. Furthermore, in Sec. IIID we investigate segmented arrays 15 of nanotubes and show that by considering also not perfectly conducting CNTs [20], in principle arbitrary potentials can be generated along the longitudinal axis of the nanotube. As an outlook, we sketch potential applications and the description of long-range interactions between two image states in Sec. IV]

\section{CYLINDRICAL IMAGE STATES ABOVE NANOWIRES}

As for charged particles in the vicinity of flat metallic surfaces, the method of images 21 can be applied in order to describe the attractive force between an infinitelylong hollow metallic cylinder of radius $a$ and a charge $q$ at $\mathbf{r}_{0}=\left(\rho_{0}>a, z_{0}, \varphi_{0}\right)$. The polarization of the cylindrical surface can be described by the induced scalar potential $\Phi_{\text {ind }}$ at $\mathbf{r}=(\rho, z, \varphi)$ via an expansion in terms of regular and irregular Bessel functions [22, $K_{\alpha}$ and $I_{\alpha}, \alpha \in \mathbb{Z}$, respectively: 


$$
\begin{aligned}
& \Phi_{\text {ind }}\left(\mathbf{r}, \mathbf{r}_{0}\right)=-\frac{2 q}{\pi} \sum_{\alpha=-\infty}^{\infty} \int_{0}^{\infty} d k \cos \left[k\left(z-z_{0}\right)\right] \\
& \times \exp \left[i \alpha\left(\varphi-\varphi_{0}\right)\right] \frac{I_{\alpha}(k a)}{K_{\alpha}(k a)} K_{\alpha}\left(k \rho_{0}\right) K_{\alpha}(k \rho) .
\end{aligned}
$$

Evaluated at the position of the external charge $\mathbf{r}=\mathbf{r}_{0}$, this yields the image potential which the charge feels:

$$
\begin{aligned}
V_{\mathrm{im}}\left(\rho_{0}\right) & =\left.\frac{q}{2} \Phi_{\text {ind }}\right|_{\left(\rho_{0}, 0,0\right)} \\
& =-\frac{q^{2}}{\pi} \sum_{\alpha=-\infty}^{\infty} \int_{0}^{\infty} d k \frac{I_{\alpha}(k a)}{K_{\alpha}(k a)} K_{\alpha}^{2}\left(k \rho_{0}\right) .
\end{aligned}
$$

Asymptotic forms of this potential were derived and an analytical expression was informed by the asymptotics [9]. In most studies thereafter, the analytical form was used in calculations in order to reduce numerical costs. Recently it was pointed out [20] that quantitative differences in eigenenergies and eigenstates existed, using the exact, cf. Eq. (2), and approximate [9] potentials, respectively.

Introducing the repulsive angular momentum barrier, in the absence of external fields, the one-dimensional effective potential reads

$$
V_{\ell}\left(\rho_{0}\right)=V_{\mathrm{im}}\left(\rho_{0}\right)+\frac{\ell^{2}-1 / 4}{2 m \rho_{0}^{2}},
$$

the parameter $\ell$ being the angular momentum and $m$ denoting the mass of the charged particle, in the following assumed to be an electron. Hereafter, we use atomic units, i.e. $\hbar=m_{e}=e=\left(4 \pi \varepsilon_{0}\right)^{-1}=1$. Whether or not detached bound states exist primarily depends on the angular momentum quantum number. For too low $\ell$ the effective potential is overall attractive everywhere and for too high $\ell$ there is no detached local potential minimum accompanying the angular momentum barrier. In earlier works $\ell_{\min }=5$ was already found to be the minimum angular momentum which supports bound TIS [20] around infinite nanowires.

The total electronic wavefunction separates in cylindrical coordinates $(\rho, z, \varphi)$,

$$
\Psi_{\ell n k}(\rho, z, \varphi)=\frac{\psi_{\ell n}(\rho)}{\sqrt{2 \pi \rho}} e^{i \ell \varphi} \phi_{k}(z),
$$

where $\rho=0$ refers to the center of the tube. This allows us to express the total energy as a sum of transverse and longitudinal energies, $E_{\ell n k}=E_{\ell n}+E_{k}$, where the transverse part scales for fixed $n$ as $E_{\ell n} \propto \ell^{-3}[9]$. Typical binding energies range between $15 \mathrm{meV}$ and a few meV.

The radial wavefunction $\psi_{\ell n}(\rho)$ satisfies the radial Schrödinger equation

$$
\left(\frac{\partial^{2}}{\partial \rho^{2}}+2\left[E_{\ell n}-V_{\ell}(\rho)\right]\right) \psi_{\ell n}(\rho)=0 .
$$

States with the same radial quantum number $n$, i.e. number of nodes of $\psi_{\ell n}$, and different $\ell$ are non-degenerate and are, in general, energetically farther apart than adjacent states of same $\ell$ but different $n$. The maximum electronic density is found at a distance $\rho_{\max }$ from the CNT, which scales as $\propto \ell^{3}[9]$. This makes a certain range of angular momenta $\ell \gtrsim \ell_{\min }$ especially interesting for our purposes since farther away from the surface the states are experimentally hard to protect against extraneous influence by the surrounding medium [11].

\section{CONTROL OF IMAGE STATES}

TISs may be controlled by means of external fields. However, these do not serve as the only control parameters for the states. Furthermore, the radius of the nanotube can be adjusted by rolling up a graphene sheet accordingly [23. So far, studies have focused on $(10,10)$ armchair nanotubes $(a=0.68 \mathrm{~nm})$. Tubes with smaller (larger) diameters tend to support states with smaller (greater) spatial extent. Moreover, the length and conducting properties may alter the TIS properties as well.

\section{A. Impact of magnetic and electric fields}

Tubular image states can be strongly affected by external magnetic fields applied parallel to the longitudinal axis $z$ [12. Neglecting any contributions stemming from the electron's spin due to its global character, two additional terms, $H_{Z}$ and $H_{d}$, a Zeeman and a diamagnetic term, respectively, enter the Hamiltonian and thus the Schrödinger Eq. (5),

$$
\begin{gathered}
H_{Z}=-\frac{B}{2} L_{z}, \\
H_{d}=\frac{B^{2}}{8} \rho^{2},
\end{gathered}
$$

where $L_{z}$ denotes the $z$-component of the angular momentum operator. For TISs, being highly extended, the diamagnetic term can dominate. Higher- $\ell$ states are farther away from the tube's surface, e.g. $\langle\rho\rangle_{\ell=5}^{n=1} \approx 7 \mathrm{~nm}$ and $\langle\rho\rangle_{\ell=7}^{n=1} \approx 18 \mathrm{~nm}$ for the $n=1$ ground states of a $(10,10)$ CNT of radius $a=0.68 \mathrm{~nm}$. Hence, states possessing higher angular momenta are more strongly affected by the presence of a magnetic field. The respective ground states of Eq. (5) are shown for $\ell=5, \ldots, 9$ in Fig. 1(a). For $\ell=5$, the states with or without an external magnetic field of $B=20 \mathrm{~T}$ essentially coincide whereas the 

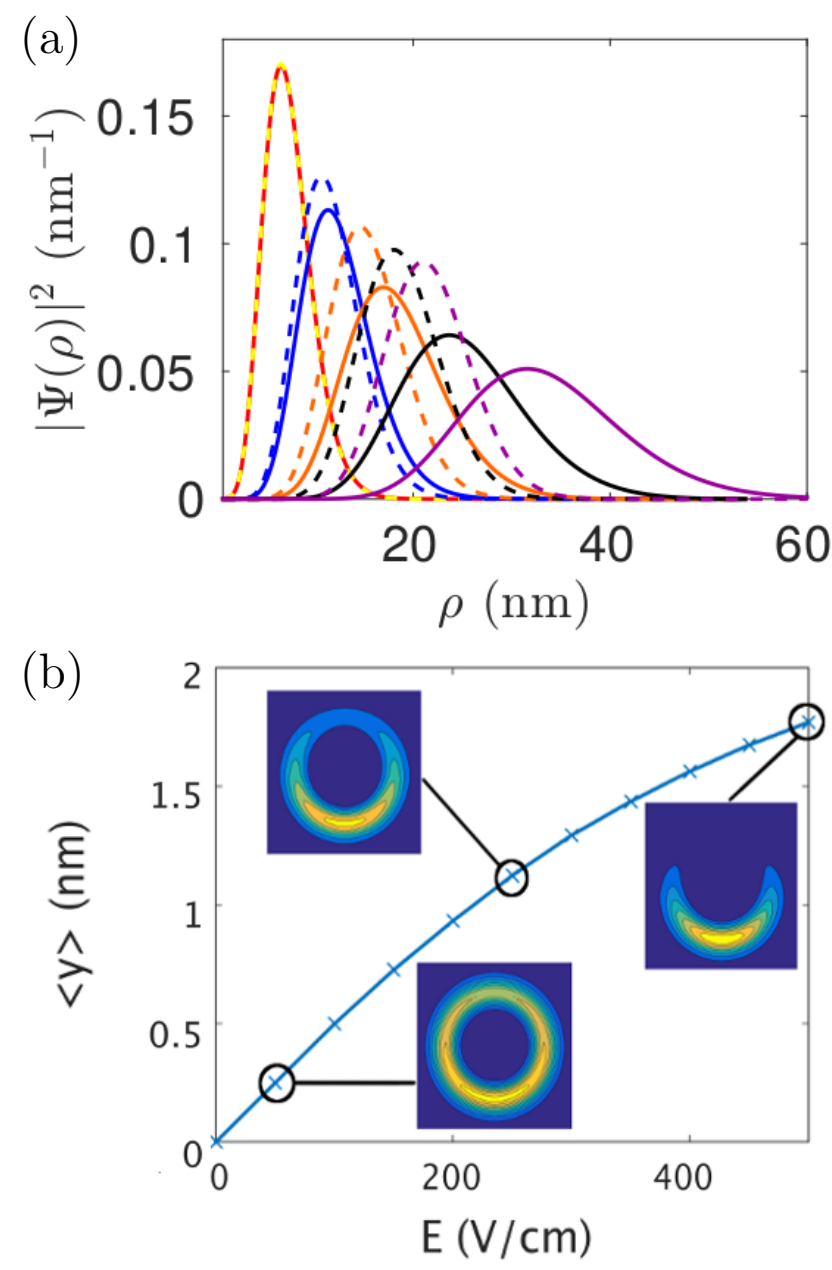

FIG. 1. (a) Radial wavefunctions $\psi_{\ell 1}(\rho)$ for angular momenta $\ell=5-9$ (from left to right) in the case of no external fields (solid) and with a magnetic field of $B=20 \mathrm{~T}$ applied (dashed) parallel to the longitudinal axis of the nanotube. (b) Mean displacement $\langle y\rangle$ perpendicular to the tube for an electric field applied along the $y$-axis for a $(l=5, n=1)$-state. The insets show the probability densities of the perturbed states for the field strengths $\mathrm{E}=50 \mathrm{~V} / \mathrm{cm}, 250 \mathrm{~V} / \mathrm{cm}$ and $500 \mathrm{~V} / \mathrm{cm}$, respectively. White dots indicate the nanotube.

higher- $\ell$ states differ significantly. Also, the spectrum is altered, again more significantly for higher-lying states.

Under the application of external electric fields perpendicular to the longitudinal axis of the nanotube, TISs lose their rotational symmetry, as states of different $\ell$ mix. The consequent electric-field induced decentering and distortion of the image states makes the position of maximum probability density vary with the field strength E, cf. Fig. 1(b). This decentering is reminiscent of a fieldinduced electric dipole. Longitudinally confined states can be selectively addressed by an external electric field. Hence, electric fields can be exploited to tune the interaction between two image states.

\section{B. Impact of finite-length segmentation}

The wavefunctions in Eq. (4) reflect that the external electron is spatially not confined along the longitudinal axis $z$. In order to induce a confining potential along $z$, we make use of the versatile electronic properties of CNTs which, above all, depend on the geometry of the CNT. Finite CNT segments with different electronic properties can be merged into a single CNT of finite length which inherits its conducting properties locally from its constituents. Moreover, the individual segments can differ in length and diameter. Periodic arrays of such finite-sized metallic nanotubes have been investigated with a focus on Bloch states in systems of identical segments connected by junctions [15]. These junctions are constructed by a rotationally asymmetric series of pentagon-heptagon defects in the lattice structure [24, 25]. In contrast, we explore CNTs consisting of non-periodically arranged segments which eventually give rise to confinement potentials for the external electron along the axis of the nanotube. In a next step, we develop a scheme that allows the preparation of several such excited states around one suspended CNT, which in turn eventually may consist of a series of metallic, semiconducting and insulating CNT segments.

As the standard method of images in electrostatics is, for flat surfaces as for cylindrical geometries, grounded on the assumption of an infinitely extended metal [21, the attractive portion of the potential 1 , binding the electron to a CNT of finite length, has to be computed differently. Since the external electron induces a charge distribution on the nanotube surface with which it interacts, the calculation of the image potential in the presence of a finite CNT reduces to the determination of the induced surface charge density. A discretized charge density, consisting of $N=10^{3}$ to $N=10^{4}$ tile charges $\left\{q_{i}\right\}_{1 \leq i \leq N}$ at positions $\left\{\mathbf{r}_{j}\right\}_{1 \leq j \leq N}$ on the surface, distributed along and around the $\mathrm{CNT}$, yields the image potential [15] as a sum over Coulombic interactions between the external electron and the tile charges:

$$
V_{\mathrm{im}}\left(\mathbf{r}_{0}\right)=-\frac{1}{2} \sum_{i=1}^{N} \frac{q_{i}}{\left|\mathbf{r}_{0}-\mathbf{r}_{i}\right|}
$$

The effective potential in Eq. (3) is thus the sum of the above potential and the centrifugal barrier potential. We are now interested in the numerical solutions of the wavefunctions $\chi_{\ell n}(\rho, z)$ from Eq. (8). Due to the longitudinal confinement which couples $\rho$ - and $z$-motion, they now depend on both $\rho$ and $z$ and are labeled by $\ell$ and $n$, a quantum number counting the energy levels. The Schrödinger equation,

\footnotetext{
${ }^{1}$ For the sake of simplicity, we will call the attractive part of the potential also the image potential for finite CNTs.
} 


\begin{tabular}{c|cccc}
$L_{\mathrm{CNT}} \ell$ & 5 & 6 & 7 & 8 \\
\hline $0.2 \mu \mathrm{m}$ & -2.8 & & & \\
$0.4 \mu \mathrm{m}$ & -7.1 & -1.9 & -0.4 & \\
$0.8 \mu \mathrm{m}$ & -10.3 & -3.9 & -1.7 & -0.7 \\
$1.6 \mu \mathrm{m}$ & -13.5 & -6.9 & -4.2 & -2.3 \\
$\infty$ & -14.0 & -7.7 & -4.8 & -3.3
\end{tabular}

TABLE I. Binding energies of degenerate bound states for $(10,10)$ nanotubes of various lengths in meV. Blank cells indicate that no bound states exist. Our $L_{\mathrm{CNT}}=\infty$ results agree up to less than a percent with [20].

$$
\left[-\frac{1}{2}\left(\frac{\partial^{2}}{\partial \rho^{2}}+\frac{\partial^{2}}{\partial z^{2}}\right)+V_{\ell}(\rho, z)-E_{\ell n}\right] \chi_{\ell n}(\rho, z)=0
$$

can be solved, e.g., by employing a two-dimensional finite differences method [26] or by setting up a discrete variable representation [27].

Since the gapped excitation spectrum of longitudinal excitations features much smaller transition energies than radial excitations, an adiabatic approximation in $z$ may be employed, reducing the numerical efforts of solving Eq. (8) to the evaluation of two one-dimensional eigenvalue equations [15]. Since the primary computational cost stems from the calculation of the surface tile charges and thus the image potential, i.e. Eq. (7), whether or not the adiabatic approximation is employed is of minor importance. Therefore, we have in most calculations solved the full problem of Eq. (8), because especially for higherlying states the resulting binding energies of the exact and approximative calculations do differ up to a few percent.

As to be expected, the image potential becomes more attractive as the length of the CNT, $L_{\mathrm{CNT}}$, increases, cf. Fig. 2(a). In the depicted range, i.e. $5 \mathrm{~nm} \leq \rho<40 \mathrm{~nm}$, the finite-tube results converge to the image potential of an infinite nanowire as $L_{\mathrm{CNT}}$ is increased. Of course, in the long-range limit $\rho \rightarrow \infty$ the electron feels the difference in attraction no matter the length of the nanotube. The lowest-lying bound states of Eq. (5) extend to less than $50 \mathrm{~nm}$ away from the tube's surface. Hence, finite-size effects affect the image potential considerably for moderate lengths $L_{\mathrm{CNT}} \lesssim 1 \mu \mathrm{m}$. The binding energies of the resulting TIS are also profoundly affected especially in this length regime. This is demonstrated in Table I for the respective $n=1$ ground states. The usual $\ell^{-3}$ scaling of the binding energies with the angular momentum is, for high- $\ell$ states, distorted by finitesize effects, since these states are to be expected far outside and therefore witness the finite spatial extent of the CNT. Note that finite geometries lead to edge effects that are also present here. Therefore, for the potential curves in Fig. 2(a), $V_{\mathrm{im}}(\rho, z)$ is evaluated at $z=L_{\mathrm{CNT}} / 2$,
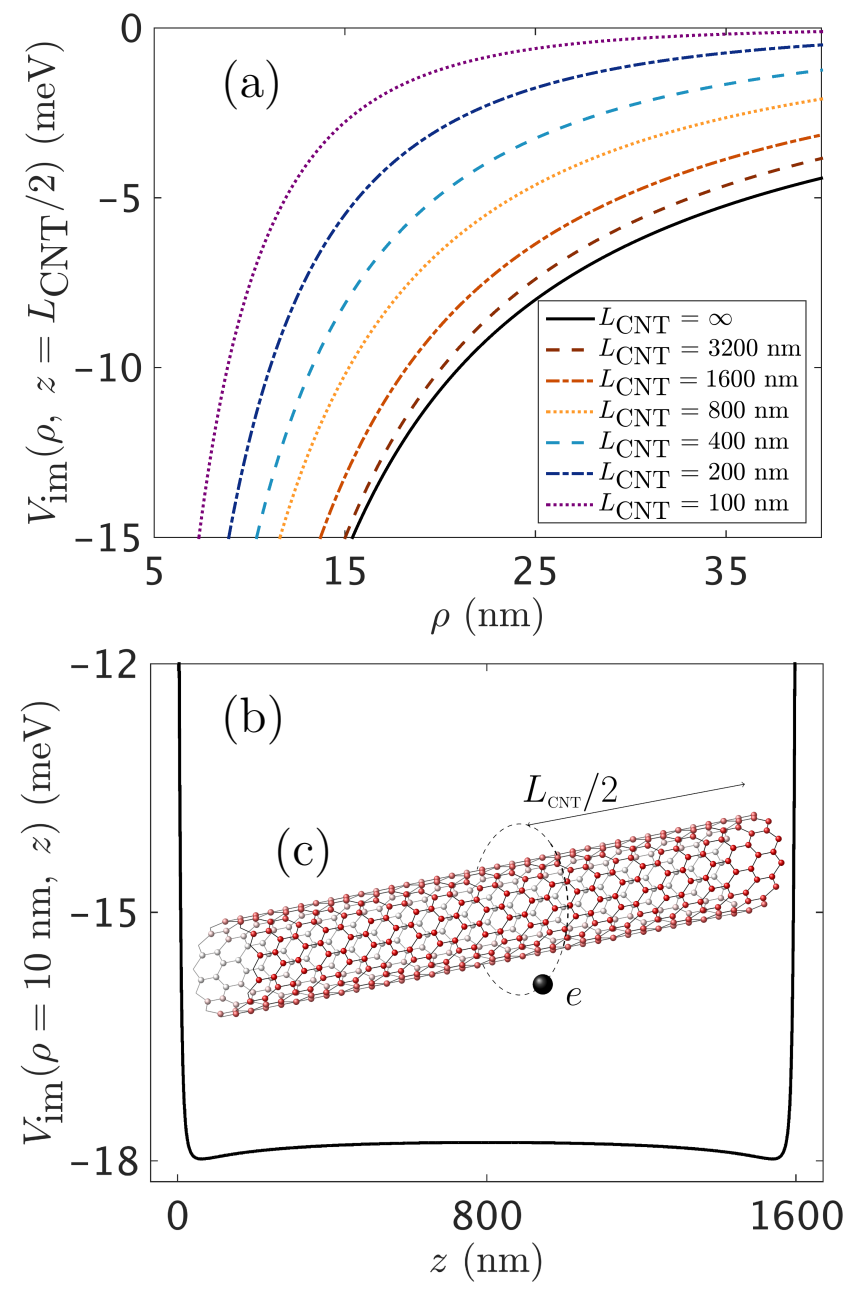

FIG. 2. (a) Image potentials for $(10,10)$ carbon nanotubes of various lengths. The potential is evaluated at $z=L_{\mathrm{CNT}} / 2$ along the tube, where $L_{\mathrm{CNT}}$ is the length of the respective nanotube. (b) Image potential at $\rho=10 \mathrm{~nm}$ and evaluated along the longitudinal axis $z$ for a $(10,10)$ carbon nanotube of length $L_{\mathrm{CNT}}=1600 \mathrm{~nm}$. (c) Sketch of CNT of length $L_{\mathrm{CNT}}$. The electronic orbit at $z=L_{\mathrm{CNT}} / 2$ shows where along the CNT the potential curves in (a) are evaluated.

cf. Fig. 2(c). Around this value, $V_{\mathrm{im}}$ is symmetric, i.e. $V_{\mathrm{im}}\left(\rho, L_{\mathrm{CNT}}^{-} / 2-z\right)=V_{\mathrm{im}}\left(\rho, L_{\mathrm{CNT}} / 2+z\right)$. Fairly long nanotubes almost generate box potentials, with shallow wells at the ends, along their axes at radial distances where the image-potential states are most likely to be found, cf. Fig. 2(b). In the presence of a single segment, the energetically lowest-lying states tend to be localized at the ends of the segment. An intuitive understanding of this can be obtained by looking at the induced charge distribution on the CNT. This charge distribution will be symmetric about the center of the tube if the external electron is located above the center of the CNT. Therefore, on both sides of the electron in longitudinal directions, charges drag the electron towards the edges. The longitudinal components of these forces cancel. In 


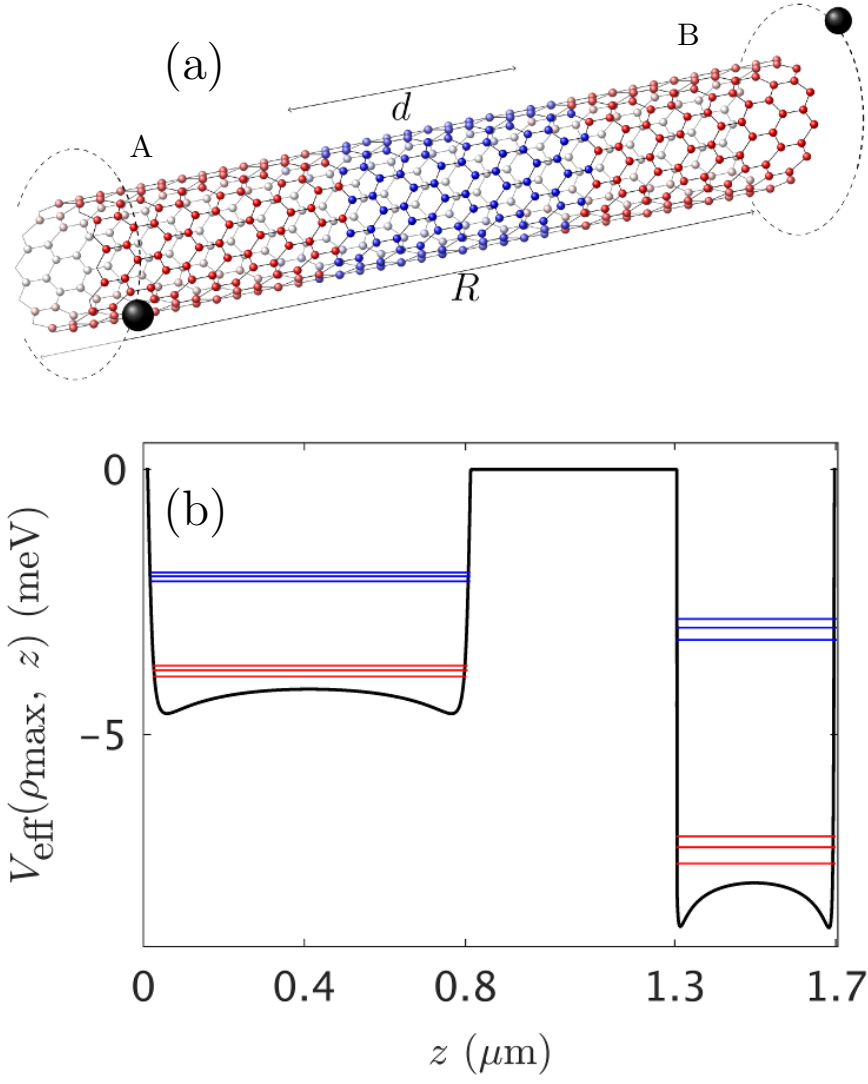

FIG. 3. (a) Scheme of three-segment nanotube with two confined electrons in tubular image states. $d$ is the length of the insulating segment that connects two metallic segments at whose ends the states are localized. The distance $R$ denotes the mean-value distance of the electronic orbits. (b) The corresponding effective potential is evaluated along $z$ at the radial distance of maximum probability density. Energy levels of the first three single-electron eigenstates of even parity $(n=1,3,5)$ in the radial ground states (red) as well as the first three single-electron eigenstates of even parity in the first-excited (blue) radial-state manifolds are shown for both subsystems.

contrast, if the electron is located closer to one of the edges, the induced charge density is also located closer to this edge and as the external electron approaches the edge, the induced charge distribution will be localized at this edge. Therefore, at fixed radial distance from the CNT, there is a total attraction towards the edges. A perfectly symmetric image potential around $z=L_{\mathrm{CNT}} / 2$ yields nearly degenerate eigenstates of even and odd parity, respectively, which are delocalized states with significant amplitude only near the edges, spread towards the center of the CNT segment for excited states [15].

\section{Localization of multiple excited states}

An electron above a non-metallic surface does either not feel the feedback of the material in form of an induced image potential at all, if the material is insulating, or sees a weaker image potential than in the metallic case, depending on the precise electronic properties of the semi-conducting CNT. Therefore, by connecting two conducting segments by an insulator, two TISs can be spatially separated, cf. Fig. 3(a). In this way, the distance between two states can be controlled a priori via the length of the insulating segment. In Fig. $3(\mathrm{~b})$, the effective potential $V_{\text {eff }}^{\mathrm{AB}}\left(\mathbf{r}_{\mathrm{A}}, \mathbf{r}_{\mathrm{B}}\right) \approx V_{\text {eff }}^{\mathrm{A}}\left(\mathbf{r}_{A}\right)+V_{\text {eff }}^{\mathrm{B}}\left(\mathbf{r}_{B}\right)$ as a function of $z$ at the radial distance of maximum probability $\rho=\rho_{\max }$ is shown for a system consisting of two spatially separated electrons both in TISs. Along the insulating segment, the effective potential is set to zero. The two external electrons are confined around metallic segments of $L_{\mathrm{CNT}}^{\mathrm{A}}=0.8 \mu \mathrm{m}$ and $L_{\mathrm{CNT}}^{\mathrm{B}}=0.4 \mu \mathrm{m}$, respectively, with angular momenta $\ell_{\mathrm{A}}=6, \ell_{\mathrm{B}}=5$. The distance $R$ between the electronic orbits is, most of all, dictated by the length $d=0.5 \mu \mathrm{m}$ of the insulating and the lengths of the metallic segments, since these states have significant amplitudes only near the edges. Also, Fig. 3(b) shows the energy levels of the three lowestlying even parity one-electron states in both the radial ground and first excited state, respectively. The odd and even parity states lie energetically so close that they cannot be resolved in Fig. 3(b). Initializing a single-electron state at one of the edges of its segment in a superposition $|L\rangle_{\sigma}=1 / \sqrt{2}\left(|0\rangle_{\sigma}+|1\rangle_{\sigma}\right)$ or $|R\rangle_{\sigma}=1 / \sqrt{2}\left(|0\rangle_{\sigma}-|1\rangle_{\sigma}\right)$, $\sigma=$ A, B labeling the two TISs around their segments and $|0\rangle_{\sigma}\left(|1\rangle_{\sigma}\right)$ denoting the corresponding even (odd) parity state, the interaction with a second TIS can be altered significantly since thereby, the mean interelectronic distance $R$ can be controlled. Since the odd and even parity states lie energetically very close, the lifetimes of the $|L\rangle_{\sigma}$ and $|R\rangle_{\sigma}$ states are comparable with the estimated lifetimes due to other decay channels.

The states can also be manipulated a priori by inserting semi-conducting segments. For finite dielectric constant $\varepsilon$, i.e. the low-frequency limit of the permittivity $\varepsilon(\omega)$, the leading-order correction to the image potential in Eqs. (2) and (7) scales as $1 / \varepsilon$ [28. Because of the sparse literature available on dielectric functions of CNTs in the static limit 29], a two-fluid model [30] was applied to infinitely long CNTs with the aim of finding a way to properly describe the image potentials due to semi-conducting CNTs 20]. However, for the sake of simplicity, we have calculated image potentials of semiconducting CNTs in the present work by assuming a macroscopically defined dielectric constant. For CNTs, it might be obtained from projecting the dielectric tensor of graphite onto a cylinder [31]. The smaller the dielectric constant is, the fainter is the attractive inward force on the electron due to the image potential. It has been remarked 20] that the band gap of a semi-conducting CNT should not exceed a value of $0.1 \mathrm{eV}$ in order to sup- 
port bound states in the effective potential. Ultimately, the set of techniques for tuning TIS mentioned so far allows for preparing the electrons in specific states along a CNT and, in the presence of external fields, with specific permanent dipole moments which can lead to controlled long-range interactions between two ore more image states [32].

\section{Engineering of image potentials}

The electronic properties of carbon nanotubes are determined by their chiral indices $(n, m)$ 33, 34. Therefore, the dielectric constants vary from nanotube to nanotube. Hence, by merging different CNTs, step-wise potentials can be generated along the longitudinal axis of a segmented CNT. The dielectric constants of the individual segments act as scaling factors for the attractive part of the potential which is thus varied along the CNT from segment to segment. The only limiting factor for generating arbitrary step-wise potentials along $z$ is the availability of CNTs with the desired electronic properties. However, nanotubes do possess versatile electronic properties and these may even be altered by doping techniques [35. Two nanotubes can be fused by imprinting pentagonheptagon defects into the lattice structure 24, 25, which serve as junctions between both tubes. To a certain degree, the chiral indices of the intial nanotube segments determine the conductivity of the joint CNT which inherits the electronic properties from its constituents. In addition, the relative rotational symmetry of the junction plays a crucial role. The rotational symmetry of a CNT is related to the lines of allowed $\mathbf{k}$ vectors in the Brillouin zone, and the rotational symmetry of the junction refers to how the pentagon-heptagon defects and hexagons are ordered along the circumference of the nanotube. Now considering a scattering process of a surface electron from one segment to another, the outcome of this strongly depends on the relative rotational symmetries of the segments as well as on the rotational symmetry of the junction. For example, a rotationally symmetric junction cannot mediate between two electronic surface states of different symmetries due to energy and angular momentum conservation laws 36]: it cannot impart any additional angular momentum to the initial state which is then totally reflected at the junction. Therefore, depending on how two CNT segments are connected, the whole nanotube may feature different electronic properties.

To illustrate the influence of the junctions' symmetries on the image potential of a series of joint CNT segments, Fig. 4 shows two image potentials of the same nanotubes but connected with different junctions. Twenty segments, all $20 \mathrm{~nm}$ in length, are stitched together by two distinct sorts of junctions: In the first case, segments with identical rotational symmetry properties, connected by a junction with a rotational symmetry mismatch relative to the segments it merges, constitute a nanotube which is overall not conducting. The resulting effective
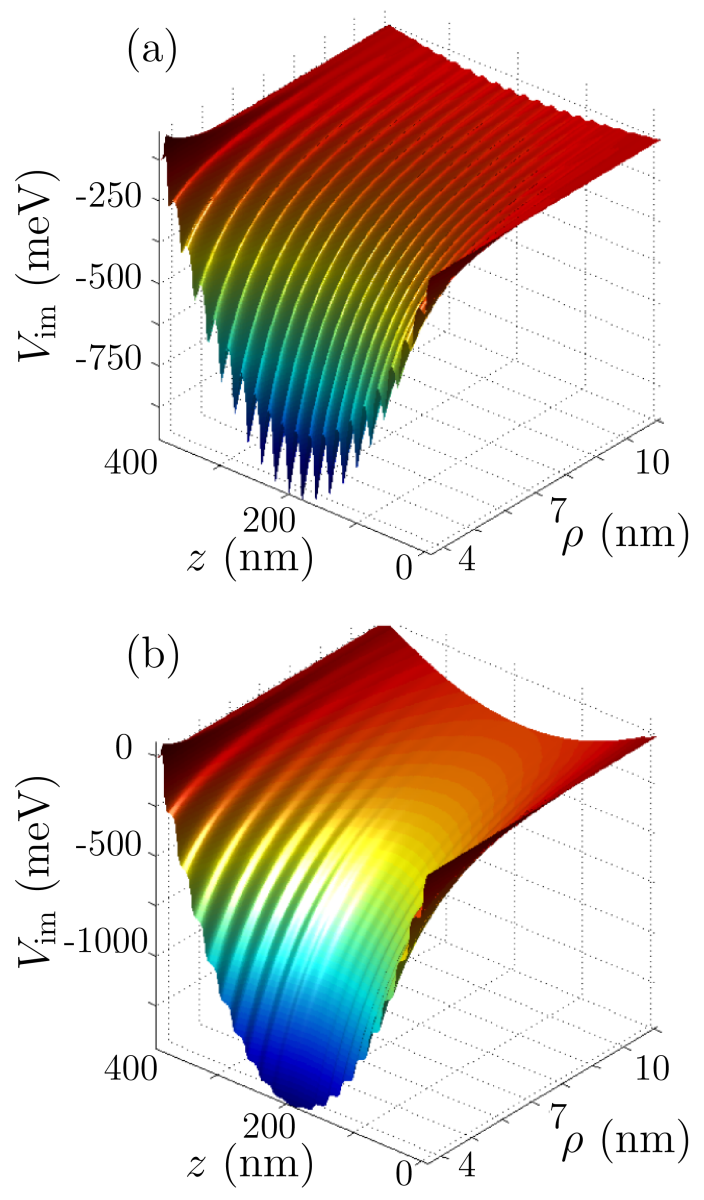

FIG. 4. Image potential $V_{\mathrm{im}}(\rho, z)$ due to a carbon nanotube of length $L_{\mathrm{CNT}}=400 \mathrm{~nm}$ consisting of twenty identical 20-nmlong segments fused by (a) rotationally asymmetric or (b) rotationally symmetric junctions, respectively.

potential $V_{\text {eff }}$ will not be smooth but feature ripples, cf. Fig. 4(a). In the second case, an almost smooth potential is the outcome as the junctions possess the same symmetry as the semi-conducting segments. At fixed radial distance $\rho$, the resulting potential is of approximately harmonic nature, cf. Fig. 4(b), and yields almost equidistantly spaced energy levels. The potentials were calculated by adjusting the dielectric constants of the individual segments accordingly in Eq. (7).

\section{SUMMARY AND OUTLOOK}

In summary, we have shown how multiple excited image states can be confined and arranged along a composite nanotube, comprised of non-periodically aligned finite nanotube segments. A framework for creating highly adjustable image potentials has been proposed, widening the range of potential control mechanisms for these exotic states. This work paves the way for subsequent studies of many-electron systems around CNTs and their interac- 
tions. The Hamiltonian of a bipartite system consisting of two image states can be represented as a sum of two isolated image-state Hamiltonians and their mutual interaction which contains the electrostatic interaction $V$, expressed via the two-body density $\hat{\rho}^{\mathrm{AB}}$ as

$$
V=\int \frac{\hat{\rho}^{\mathrm{AB}}\left(\mathbf{r}-\mathbf{r}^{\prime}\right)}{\left|\mathbf{r}-\mathbf{r}^{\prime}\right|} \mathrm{d}^{3} \mathbf{r d}^{3} \mathbf{r}^{\prime}
$$

Spin-dependent electron exchange and magnetic dipoledipole interactions may be considered. We expect strong TIS-TIS interactions at distances $<1 \mu \mathrm{m}$. The $R$ dependent interaction energy to second order can be computed efficiently with the wavefunctions in hand. We plan to extend these studies to interacting TISs, spinorbit interaction, and design of practical tube settings. Image states on liquid helium have already been demonstrated as manipulable and strongly interacting sets of qubits [16, 17]. Their versatility, especially the rather long lifetimes and moderate binding energies, but also their tunability makes TIS advantageous candidates for quantum information applications.

\section{ACKNOWLEDGMENTS}

The authors thank D. Segal for valuable comments as well as S. Krönke and S. Markson for helpful remarks on this manuscript. J. K. and P. S. gratefully acknowledge support by the National Science Foundation through a grant for the Institute for Theoretical Atomic, Molecular and Optical Physics at Harvard University and Smithsonian Astrophysical Observatory. J. K. acknowledges financial support by the German Academic Exchange Service and C. F. is grateful for support by the Studienstiftung des Deutschen Volkes in the framework of a scholarship.
[1] P. M. Echenique and J. B. Pendry, J. Phys. C: Solid State Phys. 11, 2065 (1978).

[2] P. M. Echenique and J. B. Pendry, Surf. Sci. 247, 125 (1990).

[3] P. M. Echenique, J. M. Pitarke, E. V. Chulkov, and V. M. Silkin, J. Electr. Spectr. Rel. Phen. 126, 163 (2002).

[4] S. Bose, V. M. Silkin, R. Ohmann, I. Brihuega1, L. Vitali1, C. H. Michaelis, P. Mallet, J. Y. Veuillen, M. A. Schneider, E. V. Chulkov, et al., New J. Phys. 12 (2010).

[5] U. Höfer, I. L. Shumay, C. Reu, U. Thomann, W. Wallauer, and T. Fauster, Science 277, 1480 (1997).

[6] S. Link, H. A. Dürr, G. Bihlmayer, S. Blügel, W. Eberhardt, E. V. Chulkov, V. M. Silkin, and P. M. Echenique, Phys. Rev. B 63, 115420 (2001).

[7] W. Berthold, U. Höfer, P. Feulner, E. V. Chulkov, V. M. Silkin, and P. M. Echenique, Phys. Rev. Lett. 88, 056805 (2002).

[8] M. W. Cole and M. H. Cohen, Phys. Rev. Lett. 23, 1238 (1969).

[9] B. E. Granger, P. Král, H. R. Sadeghpour, and M. Shapiro, Phys. Rev. Lett. 89, 135506 (2002).

[10] G. Gumbs, A. Balassis, A. Iurov, and P. Fekete, The Scientific World Journal 2014, 726303 (2014).

[11] M. Zamkov, N. Woody, S. Bing, H. S. Chakraborty, Z. Chang, U. Thumm, and P. Richard, Phys. Rev. Lett. 93, 156803 (2004).

[12] D. Segal, P. Král, and M. Shapiro, Surf. Sci. 577, 86 (2005).

[13] M. Zamkov, H. S. Chakraborty, A. Habib, N. Woody, U. Thumm, and P. Richard, Phys. Rev. B 70, 115419 (2004).

[14] D. Segal, B. E. Granger, H. R. Sadeghpour, P. Král, and M. Shapiro, Phys. Rev. Lett. 94, 016402 (2005).

[15] D. Segal, P. Král, and M. Shapiro, Phys. Rev. B 69, 153405 (2004).

[16] P. M. Platzman and M. I. Dykman, Science 284, 1967 (1999).

[17] M. Dykman and P. Platzman, Fortschritte der Physik
48, 1095 (2000).

[18] D. Segal, P. Král, and M. Shapiro, Chem. Phys. Lett. 392, 314 (2004).

[19] D. Segal, P. Král, and M. Shapiro, J. Chem. Phys. 122, 134705 (2005).

[20] S. Segui, C. Celedón López, G. A. Bocan, J. L. Gervasoni, and N. R. Arista, Phys. Rev. B 85, 235441 (2012).

[21] J. D. Jackson, Classical electrodynamics (Wiley, New York, NY, 1999), 3rd ed.

[22] N. R. Arista and M. A. Fuentes, Phys. Rev. B 63, 165401 (2001).

[23] M. Terrones, Ann. Rev. Mat. Res. 33, 419 (2003).

[24] J.-C. Charlier, T. W. Ebbesen, and P. Lambin, Phys. Rev. B 53, 11108 (1996).

[25] L. Chico, V. H. Crespi, L. X. Benedict, S. G. Louie, and M. L. Cohen, Phys. Rev. Lett. 76, 971 (1996).

[26] R. LeVeque, Finite Difference Methods for Ordinary and Partial Differential Equations: Steady-State and TimeDependent Problems (Society for Industrial and Applied Mathematics, 2007).

[27] J. C. Light and T. Carrington, Adv. Chem. Phys 114, 263 (2000).

[28] J. L. Gervasoni and N. R. Arista, Phys. Rev. B 68, 235302 (2003).

[29] W. Lu, D. Wang, and L. Chen, Nano Lett. 7, 2729 (2007).

[30] D. J. Mowbray, S. Segui, J. Gervasoni, Z. L. Mišković, and N. R. Arista, Phys. Rev. B 82, 035405 (2010).

[31] T. Stöckli, Z. L. Wang, J.-M. Bonard, P. Stadelmann, and A. Châtelain, Phil. Mag. B 79, 1531 (1999).

[32] M. Saffman, T. G. Walker, and K. Mølmer, Rev. Mod. Phys. 82, 2313 (2010).

[33] R. Saito, G. Dresselhaus, and M. S. Dresselhaus, Physical Properties of Carbon Nanotubes (World Scientific Publishing, 1998).

[34] J.-C. Charlier, X. Blase, and S. Roche, Rev. Mod. Phys. 79, 677 (2007).

[35] X. Li, L. M. Guard, J. Jiang, K. Sakimoto, J.-S. Huang, J. Wu, J. Li, L. Yu, R. Pokhrel, G. W. Brudvig, et al., 
Nano Lett. 14, 3388 (2014).

[36] L. Chico, L. X. Benedict, S. G. Louie, and M. L. Cohen, Phys. Rev. B 54, 2600 (1996). 